\title{
Espacios y emociones: una propuesta desde la ontología histórica*
}

Juan Manuel Zaragoza Bernal

\section{Facultad de Filosofía (Universidad de Murcia)}

jm.zaragozabernal@um.es - https://orcid.org/0000-0001-8377-6688

Fecha recepción: 06.05.2020 / Fecha aceptación: 22.12.2020

\section{Resumen}

En este artículo proponemos una nueva aproximación al estudio de las emociones desde una aproximación transdisciplinar a la historia y a la filosofía. Para ello, cuestionamos lo que llamamos teoría del appraisal y apostamos por una definición social de las emociones, basada en la teoría de la emoción construida de Lisa Feldman Barrett. En concreto, proponemos considerar a las emociones como categorías humanas, lo que nos permitiría su estudio

\begin{abstract}
Here, we propose a new interdisciplinary approach to research on emotions from the perspectives of history and philosophy. To this end, we question appraisal theory and advocate a social definition of emotions based on Lisa Feldman Barrett's theory of constructed emotions. Thus, following Ian Hacking, we contend that emotions are not natural, but human kinds, and propose historical ontology as our methodology. Specifically, we explore how
\end{abstract}

\footnotetext{
* Este trabajo forma parte del Proyecto de Investigación del Plan Nacional de $\mathrm{I}+\mathrm{D}+\mathrm{i}$ «Improvisación y contagio emocional. Historia y teoría de experiencias emocionales» (PID2019-108988GB-I00) del Ministerio de Ciencia e Innovación y del Programa Renovación Generacional para el Fomento de la Investigación «La naturaleza como horizonte ético. Modernidad, crisis ecológica y experiencia del orden» (RG2020-004UM) de la Universidad de Murcia.
} 
como parte de la ontología histórica. A partir de aquí, analizamos el problema acerca de la relación entre cultura material y experiencia emocional y proponemos el concepto de matriz, desarrollado por Ian Hacking, como una forma de ligar ambas. Apoyaremos esta aproximación con un estudio de caso: el del Hospital para Mujeres Incurables Jesús Nazareno, en Madrid, en la década de 1870. Este caso nos servirá para identificar y probar un conjunto de herramientas metodológicas que nos ayuden a analizar aquellos componentes materiales que construyen, determinan y condicionan nuestras experiencias emocionales.

\section{Palabras clave}

experiencia emocional, teoría de la emoción construida, clases humanas, Ian Hacking, Hospital para Mujeres Incurables Jesús Nazareno material culture and our emotional experiences are related and propose Hacking's "matrix" as an answer. Through a case study of the Jesús Nazareno Hospital for Incurable Women (Madrid, Spain) in the 1870s, we identify and test a series of theoretical concepts that facilitate an analysis of the material components that build, shape and determine our emotional experiences.

\section{Keywords}

emotional experience, theory of constructed emotions, human kinds, Ian Hacking, Jesús Nazareno Hospital for Incurable Women 


\section{Introducción: la teoría de las emociones construidas y la propuesta desde la ontología histórica.}

Podemos dividir el estudio filosófico de las emociones en dos grandes grupos. Por un lado, tendríamos aquellos que estudian las emociones en tanto que emociones ${ }^{1}$; por el otro, aquellos interesados en el pensamiento de autores concretos respecto a las emociones ${ }^{2}$. Entre los primeros podemos trazar otra división, de acuerdo a si consideran que las emociones tienen valor cognitivo ${ }^{3}, \mathrm{o} \mathrm{no}^{4}$. Ambas posturas, sin embargo, comparten una misma concepción básica de qué es una emoción, lo que podríamos llamar, de forma tentativa, la teoría de las emociones como appraisal, y que la psicóloga Lisa Feldman Barrett describe como sigue:

It is assumed that each kind of emotion can be identified by a more or less unique signature response (within the body) that is triggered or evoked by a distinct causal mechanism (within the brain). As a result, it should be possible to recognize distinct emotions in other people, identify them in oneself, and measure them in the face, physiology, and behavior ${ }^{5}$.

Esta teoría se sustentaría en una definición de las emociones como clases naturales, ya sea en un sentido laxo según el cual la «clase» se definiría a partir de un conjunto de propiedades homeostáticas ${ }^{6}$, o en el sentido fuerte según el cual aquello que llamamos emociones

1. J. J. Prinz, Gut Reactions: A Perceptual Theory of Emotion, Oxford,2004.

2. J. Dow, "Aristotle's Theory of the Emotions: Emotions as Pleasures and Pains", en M. Pakaluk y G. Pearson (eds.), Moral Psychology and Human Action in Aristotle, Oxford, 2011, 47-74.

3. M. C. Nussbaum, Upheavals of Thought: The Intelligence of Emotions, Cambridge, 2001.

4. Prinz, Gut Reactions..., op.cit.

5. L.F. Barrett, “Are Emotions Natural Kinds?", Perspectives on Psychological Science, 1, 2006: 30.

6. R. Boyd, "Realism, Anti-Foundationalism and the Enthusiasm for Natural Kinds", Philosophical Studies: An International Journal for Philosophy in the Analytic Tradition 61, 1/2, 1991: 127-48; P. E. Griffiths, What 
comparten una esencia común ${ }^{7}$. No nos interesa, en este momento, analizar estas propuestas, algo que ya hicimos en un texto anterior ${ }^{8}$, sino apuntar la aparición de una nueva teoría que entiende que las emociones son el resultado final de un complejo proceso que incluye aspectos relacionados con la química cerebral, la percepción de nuestros estados corporales (interocepción), una concepción del cerebro como red neuronal, etc., pero también, y esto es lo relevante, por elementos que llamaríamos culturales:

En pocas palabras, vemos que las emociones [...] en lugar de ser universales varían de una cultura a otra; que no son provocadas sino que las creamos nosotros; que surgen de una combinación entre las propiedades físicas del cuerpo, un cerebro flexible cuyas conexiones reflejan el entorno en el que se desarrolla, y la cultura y la educación que ofrecen ese entorno. Las emociones son reales, pero no en el mismo sentido objetivo que las moléculas o las neuronas. Son reales en el sentido en que lo es el dinero, es decir, no son una ilusión, pero sí un producto del consenso humano?.

La propuesta de Barrett nos ofrece una visión de las emociones construidas en la que los factores sociales y culturales son determinantes, no para colorear una emoción (uno de las muchas metáforas con las que se ha pretendido explicar la influencia de los factores culturales en nuestra experiencia emocional), sino para determinar qué emoción surgirá de este proceso compositivo. Las emociones, nos dice Barrett, son el resultado de un "consenso». En consecuencia, las emociones ya no son un objeto de estudio por sí mismas, sino que son reemplazadas por el concepto, más comprehensivo, de experiencia (emocional) ${ }^{10}$. Esto ofrece, a las humanidades y ciencias sociales, la posibilidad de generar proyectos de investigación innovadores, que permitan un estudio verdaderamente transdisciplinar ${ }^{11}$.

En el presente artículo presentaremos una propuesta basada en la ontología histórica de Ian Hacking. Si las emociones no son clases naturales, si, como dice Barrett, son «producto del consenso humano», ¿qué tipo de clases son? Nuestra propuesta pasa por considerarlas clases relevantes ${ }^{12}$. Las clases relevantes serían, para Hacking, aquellas que empleamos en las humanidades y en las ciencias sociales. Es decir, aquellas que aplicamos a los humanos. Su

Emotions Really Are: The Problem of Psychological Categories, Chicago, 1998.

7. Prinz, Gut Reactions..., op.cit., 102.

8. J.M. Zaragoza, "Investigación filosófica y emociones: la ontología histórica de Ian Hacking como alternativa", Daimon, in press.

9. L.F. Barrett, La vida secreta del cerebro: Cómo se construyen las emociones, Barcelona, 2018, 18.

10. Añadimos el modificador "emocional" al concepto de "experiencia" para subrayar los aspectos emocionales de la misma, tradicionalmente ignorados. Obviamente, es imposible que se dé una experiencia sin una combinación de razón y emoción. Sí es cierto, sin embargo, que a falta de aumentar nuestra comprensión acerca de cómo se construyen nuestras emociones, tal vez deberíamos poner en suspenso esta división tan estricta. Pero este es un tema que desborda, por mucho, los límites de este artículo.

11. Zaragoza, "Investigación filosófica..., op. cit.

12. N. Goodman, "Words, Works, Worlds", Erkenntnis 9, 1, 1975: 57-73; I. Hacking, "On Kripke's and Goodman's Uses of 'Grue', Philosophy 68, 265, 1993: 269-95. 
lógica y funcionamiento serían totalmente distintos al de las clases naturales, y se caracterizarían, principalmente, por su efecto bucle:

[...] as we evolve an idea about a kind of person or of human behavior, people change, behaviors change. Children experience their hurt differently. They are more self-conscious about when and how emotional and sexual abuse is painful [...] New kinds of people come into being that don't fit the wisdom just acquired, less because the recent knowledge was wrong than because of a feedback effect ${ }^{13}$.

Una de las dificultades que una teoría de las emociones como la que nos plantea Barrett debe resolver es acerca del papel que la cultura material desempeña en nuestra experiencia emocional. El concepto de «matriz», desarrollado por Hacking en su libro ¿La construcción social de qué? (2001), puede ayudarnos en esta tarea. Las ideas, nos dice Hacking, no "habitan» en el vacío, sino que se encuentran dentro de un "marco social». Este marco social es la matriz dentro de la cual el concepto se forma: el conjunto de instituciones, prácticas y agentes que dan sentido a este concepto, que lo constituye. Y entre ellos encontramos la cultura material: «ustedes pueden querer llamar sociales a estas cosas porque lo que realmente nos importa son sus significados, pero son materiales, y en su cruda materialidad establecen diferencias sustanciales entre las personas» ${ }^{14}$. También la matriz se ve afectada por cómo pensemos la categoría y esta, a su vez, es influenciada por los cambios materiales que se producen en su matriz. Y esto es así porque, en realidad, ambas son inseparables: «Esta exposición de las ideas y la clasificación da por supuesto lo que es obvio, principalmente que funcionan sólo dentro de una matriz [...] Cuando leemos sobre la construcción social de $X$, a lo que se hace referencia muy comúnmente es a la idea de $X$ (en su matriz) $»^{15}$.

La ontología histórica, a partir del concepto de «matriz», puede ayudarnos a ampliar nuestra comprensión acerca de cómo el espacio y la cultura material afectan a nuestra experiencia emocional ${ }^{16}$. Pero para ello, será primero necesario identificar y caracterizar los factores espaciales que influyen en la composición de nuestras emociones.

\section{Espacios enmarcados y emociones}

La influencia del espacio y su impacto en nuestra experiencia emocional ha recibido una atención creciente, tras el llamado giro afectivo de los años $2000^{17}$, en el ámbito de la psicolo-

13. I. Hacking, "The Making and Molding of Child Abuse", Critical Inquiry 17, 1991: 254.

14. I. Hacking, ¿La construcción social de qué?, Barcelona, 2001, 33.

15. Hacking, ¿La construcción..., 33.

16. Hacking, ¿La construcción..., 34.

17. P. T. Clough y J. O. Halley (eds.), The Affective Turn: Theorizing the Social, Durham, N.C., 2007. 
gía ${ }^{18}$, la geografía humana ${ }^{19} y$, más recientemente, en el de la historia ${ }^{20}$. En filosofía, el estudio del espacio en relación con las emociones se ha realizado, principalmente, desde la tradición fenomenológica. A partir de las investigaciones de Hermann Schmitz o Gernot Böhme se ha desarrollado un profundo trabajo alrededor del concepto de atmósfera ${ }^{21}$, sobre el que volveremos más adelante, por no hablar de la aportación de Peter Sloterdijk, que escapa a los límites de nuestro estudio ${ }^{22}$.

Nuestra propuesta, sin embargo, parte de otro lugar. Concretamente, de la metáfora dramatúrgica y el análisis de marcos de Erving Goffman ${ }^{23}$. Como ya hemos señalado en textos anteriores ${ }^{24}$ tomamos tres elementos de Goffman:

1. La idea de escena como medio material en el que la acción humana se desarrolla ${ }^{25}$.

2. El concepto de marco experiencial, que para Goffman es el conjunto de elementos culturales (objetos materiales, normas, espacios) que «convierte en algo que tiene sentido» a lo que ocurre en la escena ${ }^{26}$.

18. A. Metcalfe y A. Game, "Potential space and love", Emotion, Space and Society 1, 2008: 18-21.

19. B. Anderson, "Affective Atmospheres", Emotion, Space and Society 2, 2009: 77-81; V. L. Henderson, "Is there Hope for Anger? The Politics of Spacializing and (Re)producing an Emotion", Emotion, Space and Society 1, 2008: 28-37.

20. La literatura sobre emociones y cultura material empieza a ser bastante numerosa, aunque se trata, todavía, de un campo poco explorado, al menos comparativamente. Algunos textos fundamentales son: J. Labanyi, "Doing Things: Emotions, Affect, and Materiality", Journal of Spanish Cultural Studies 11, 3, 2010, 223 - 233; M. Pernau, "Space and Emotion: Building to Feel", History Compass 12, 2014, 541-49; J.M. Zaragoza, "Ampliar el marco. Hacia una historia material de las emociones", Vinculos de Historia, 4, 2015: 28-48; B. Gammerl, J. S. Hutta, y M. Scheer, "Feeling differently: Approaches and their politics", Emotion, Space and Society 25, 2017, 87-94; A. Moran y S. O'Brien, Love Objects: Emotion, Design and Material Culture, London \& New York, 2014; S. Holloway, The Game of Love in Georgian England: Courtship, Emotions, and Material Culture, Oxford, 2019. La popularidad de la historia de las emociones ha hecho que empiecen a aparecer diversos libros con vocación de ser "introducciones" a la nueva disciplina, y muchos de ellos incluyen referencias a los trabajos relacionados con la cultura material. Véase, por ejemplo: B. H. Rosenwein y R. Cristiani, What is the History of Emotions?, Cambridge \& Malden, 2017; R. Boddice, The History of Emotions, Manchester, 2018.

21. H. Schmitz, R. O. Müllan, y J. Slaby, "Emotions Outside the Box-the New Phenomenology of Feeling and Corporeality", Phenomenology and the Cognitive Sciences 10, 2011: 241-59; G. Böhme, The Aesthetics of Atmospheres, London; New York, 2016.

22. P. Sloterdijk, Esferas III: Espumas. Esferología plural, Madrid, 2018.

23. E. Goffman, La presentación de la persona en la vida cotidiana, Buenos Aires, 1971; E. Goffman, Frame Analysis. Los marcos de la experiencia, Madrid, 2006.

24. J.M. Zaragoza, "Espaciones emocionales y medicina en el siglo XIX. Una ontología histórica de las pasiones", Asclepio, en prensa.

25. Goffman, La presentación... op. cit., 34.

26. Goffman, Frame Analysis... op. cit., 23. 
3. El espacio marco (frame space), que vendría a ser el conjunto de posibilidades de acción significativa disponible para un actor en un momento y espacio dados ${ }^{27}$.

A partir de la conjunción de estos tres elementos proponemos un cuarto, que denominaremos espacio enmarcado (framed space), y que caracterizamos como el espacio diseñado y construido para que en su interior se desarrollen un conjunto de marcos experienciales y no otros. El ejemplo más evidente tal vez sea un templo. Los aspectos materiales del templo, desde su composición espacial hasta el empleo de sustancias aromáticas, influyen en nuestra experiencia del mismo y conforman los significados que damos a las acciones que se realizan en su interior. Esto no quiere decir que no se puedan dar otros tipos de experiencia en el templo, pero sí que éste ha sido pensado para sancionar unas sobre otras.

Se da el caso, además, de que normalmente los espacios contienen más de un marco. Así, en un parque podemos ver gente jugando al cricket, haciendo picnics familiares, reuniones religiosas, manifestaciones políticas, etc. En otros casos, sin embargo, el abanico de marcos sancionados será mucho menor. Los espacios enmarcados varían en su composición y diseño, y determinan así nuestras posibilidades de experiencia ${ }^{28}$. Cada uno de estos espacios, además, contiene un marco emocional propio, entendido como el conjunto de posibilidades de experiencia emocional disponibles en el mismo: no experimentamos las mismas emociones en un campo de fútbol y en un cementerio, por mucho que, a veces, sí lo sean las acciones realizadas (por ejemplo, llorar).

¿Qué elementos son los que conforman el marco emocional? Tomemos como ejemplo un hospital. Todos estaríamos de acuerdo en que las normas disciplinarias recogidas en su reglamento, así como las decisiones tomadas por su junta disciplinaria, serían algunos de ellos. Son elementos que resultan conocidos, y que han sido estudiados con anterioridad por el mismo Goffman, en su trabajo sobre los hospitales psiquiátricos ${ }^{29}$, o por Michel Foucault en Vigilar y Castigar y en sus trabajos sobre la gubernamentalidad ${ }^{30}$. En el presente texto nos distanciamos de este tipo de estudios para centrarnos en aquellos elementos materiales que conforman el espacio.

Si, tal y como señalamos antes, espacio y marco están relacionados, podemos entender que habrá elementos en el primero que formen parte del segundo. Es decir, que determinados elementos del espacio en que se desarrolla la acción son determinantes a la hora de interpretarla. Cuando marco y espacio se identifican, esto es, cuando la acción se hace comprensible sólo si se desarrolla en un espacio determinado, los elementos espaciales serán determinantes para tal comprensión. Goffman analiza esta relación entre acción y espacio en La presentación de la persona en la vida cotidiana:

27. E. Goffman, Forms of Talk, Oxford, 1981, 230.

28. F. Broncano, "In media res: cultura material y artefactos", Artefactos 1, 2008, 18-32.

29. E. Goffman, Asylums: Essays on the Social Situation of Mental Patients and Other Inmates, Chicago, 1961.

30. M. Foucault, Surveiller et punir: Naissance de la prisión, Paris, 1975. 
La decoración e instalaciones permanentes de un lugar en el cual se desarrolla generalmente una determinada actuación, así como los actuantes y la acción que allí solemos encontrar, tienden a imprimir en él una especie de hechizo; aun cuando la actuación habitual no se lleve a cabo allí, el lugar tiende a retener algo del carácter de su región anterior ${ }^{31}$.

Este «hechizo» que señala Goffman no es otra cosa que el efecto de los elementos espaciales y materiales que forman parte del marco experiencial contenido en la «escena». Es importante señalar, por un lado, que este «hechizo» -por seguir utilizando el término de Goffman-, resulta de la colaboración entre los sujetos que llevan a cabo la acción y el entorno material en que es llevada a cabo. No en vano el «hechizo» se imprime, a través de la interacción entre actor, actuación y espacio material. Por otro lado, el espacio adquiere cierta autonomía, ya que el «hechizo» permanece, aun cuando ni el sujeto, ni la actuación se estén llevando a cabo en ese preciso momento

Los estudios sobre esta relación entre sujeto y espacio material se han desarrollado especialmente en el campo de la arqueología, y más concretamente en lo que se ha dado en llamar arqueología post-procesual ${ }^{32}$. Dentro de este movimiento, que rechazaba tendencias objetivistas y cientifistas de corrientes anteriores, se desarrolla una línea de pensamiento que buscaba explorar la experiencia de los habitantes del pasado a través de su relación con los espacios que habitaban. Dicha corriente, de origen británico, se identificó con la fenomenología de corte husserliana, mediada por la recepción hecha a través del existencialismo de mitad del siglo XX, tanto heideggeriano como sartreano:

[the] phenomenologists try to describe what is like in human terms to walk along, around, through these monuments, what one sees and hears at different points, how one's experience is affected by temporal rhythm (the order in which one sees things, the appearance of sudden 'surprises' over the horizon.) $)^{33}$

La principal crítica que se hace a la corriente fenomenológica es que esta fracasa en su intento de entender la experiencia de los habitantes del pasado debido a un error de principio en su propuesta: la única forma de entender la experiencia pasada sería a través de nuestra propia experiencia de dichos lugares. La arqueología fenomenológica asumiría así una suerte de atemporalidad, de unidad empática de la experiencia humana que le permitiría crear un vínculo directo entre nuestra experiencia actual y la de aquellos que nos antecedieron hace miles de años. Como señalaron diversos críticos, no exentos de cierta maldad, el habitante de la edad de piedra descrito por estos estudios se parecía demasiado a un doctor en arqueología por Oxford o Cambridge ${ }^{34}$.

31. Goffman, La presentación ... op. cit., 135-36. Énfasis nuestro.

32. L. M. Hurcombe, Archaeological Artefacts as Material Culture, London \& New York, 2007, 91.

33. M. Johnson, Archaeological Theory. An Introduction, Chichester, 2010, 118.

34. Johnson, Archaeological..., op. cit., 119. 
Han sido varios los intentos de poner al día esta tradición, buscando alternativas a las acusaciones de presentismo y al uso indiscriminado de la empatía como herramienta heurística. Uno de ellos, que merece la pena destacar, es el artículo Rethinking emotions and material culture, de Harris y Sørensen, que además enfrenta el problema de las emociones y el espacio (entendido como cultura material) ${ }^{35}$. Su propuesta pasa, en primer lugar, por rechazar la interpretación de las emociones como algo puramente subjetivo e inmaterial, trasladándolas, por el contrario, al entorno socialmente constituido de la experiencia intersubjetiva $^{36}$. En segundo lugar, señalan el papel indispensable de las relaciones entre sujeto y cultura material en la constitución de las emociones:

A starting point for this is Gosden's [2005] concise conclusion that 'emotions are materially constituted and material culture is emotionally constituted'. This argument is supported by the range of work in different disciplines that now emphasizes that human beings and material things recursively shape each other. ${ }^{37}$

Finalmente, los autores proponen un vocabulario crítico que facilite el análisis del investigador a la hora de enfrentarse a los espacios y a su posible impacto emocional. En este «vocabulario analítico» encontramos influencias de una multitud de tradiciones teóricas, que incluirían a la antropología de las emociones, los estudios de cultura material del University College of London y, por supuesto, la tradición fenomenológica de la arqueología británica ${ }^{38}$. Los autores distinguen cuatro términos que hacen referencia a otros tantos aspectos discernibles por el análisis en la relación espacio-sujeto-emociones:

- emotion: the embodied act of being moved to move;

- affective fields: the networks of people and things through which emotions are generated;

- attunement: the practice of attending to the material world and its emotional qualities;

- atmosphere: the emotional experience engendered by being in a particular place and situation $^{39}$.

Los autores señalan explícita y reiteradamente que esta distinción no se basa en ningún tipo de separación fenomenológica entre los cuatro términos, ni mucho menos que exista una escala jerárquica. Su finalidad, insisten, es puramente analítica.

35. Un artículo también comentado en Rosenwein y Cristiani, What is..., op. cit.

36. O. J. T. Harris y T. F. Sørensen, "Rethinking Emotion and Material Culture", Archaeological Dialogues $17,2010,147$.

37. Harris y Sørensen, "Rethinking ..., op. cit., 147.

38. C. Lutz y G. M. White, "The anthropology of emotions", Annual review of anthropology 15, 1986, 405-436; Ch. Tilley, A Phenomenology of Landscape: Places, Paths, and Monuments, Oxford, 1994; D. Miller, Material Cultures. Why Some Things Matter, London: 1998.

39. Harris y Sørensen, "Rethinking ..., op. cit., 153. 
Desde nuestro punto de vista, el estudio de Harris y Sørensen ofrece una importante aportación al problema de cómo el espacio emocional afecta al individuo. Nos referimos, en concreto, al desarrollo de la idea de atmósfera, que definen, siguiendo a Gernot Böehme, como el resultado de la interacción entre personas, espacios y cosas. Es, en este sentido, similar al «hechizo» de Goffman, pero al combinarlo con los otros tres elementos nos proporciona una explicación de cómo dicho «hechizo» es conjurado. No estamos de acuerdo, sin embargo, con la apreciación acerca de la necesidad de que la atmósfera sea percibida para que sea efectiva. En última instancia no estamos de acuerdo con la necesidad del atunnement para que el proceso tenga lugar. El concepto, que según ellos toman de Heidegger, no haría referencia únicamente a cómo el entorno afecta a nuestra tonalidad afectiva (Stimmung), sino que para ellos es también «how people notice, observe, perceive and recognize moods and emotions in themselves and others» ${ }^{40}$. Partiendo del hecho de que, efectivamente, un reconocimiento de este tipo puede afectar a nuestra experiencia emocional, no consideramos que sea necesario para que nos veamos afectados por la atmósfera de un espacio, sino que pensamos que la función del entorno material en la constitución de marcos emocionales es más eficaz si no somos conscientes de dicho papel, en el sentido expresado por el concepto de la humildad de las cosas, del antropólogo Danny Miller ${ }^{41}$.

Volvamos a la propuesta de los autores. Al situar al sujeto dentro de un determinado espacio, este pasa a formar parte de un campo afectivo. El campo afectivo se define como una red de relaciones entre sujetos y objetos que son producidas por $-\mathrm{y}$ a su vez son productoras de- prácticas. Esta relación entre sujeto y objeto es material. Se produce como resultado del encuentro entre la materialidad del cuerpo y la materialidad del artefacto. Más concretamente, como resultado de las prácticas que los ponen en relación. En su caso, se trata de sujetos caminando entre altos troncos cortados en un emplazamiento ritual del paleolítico. El campo afectivo, pese a poder estar estrechamente ligado a un espacio concreto, es independiente de él. En cierto sentido es más extenso, pues incluye a aquellos sujetos y artefactos que, sin pertenecer de forma física al espacio, irrumpen en él desde el exterior. Por ejemplo, aspectos como las normas y códigos disciplinarios. Esta versión extendida del campo afectivo se correspondería con el marco emocional.

La atmósfera, por su parte, sería el resultado del marco emocional asociado a un lugar determinado. Si el campo afectivo sirve para etiquetar las relaciones entre sujeto y cultura material, la atmósfera introduce el elemento espacial, al situar dicha interacción en un espacio delimitado y que ha sido diseñado para contener un marco concreto, o varios, como ya hemos visto. Campo afectivo y atmósfera nos permiten analizar los complejos procesos que relacionan espacio, sujeto y objeto, entre cuyos resultados se encuentra la aparición, más o menos controlada, de emociones.

40. Harris y Sørensen, “Rethinking ..., op. cit., 151.

41. D. Miller, Artefact as Categories. A Study of Ceramic Variability in Central India, Cambridge, 1985, 181. 


\section{La atmósfera del Hospital para Mujeres Incurables Jesús Nazareno de Madrid}

¿Cómo aplicaríamos estas consideraciones al análisis de un espacio y, en concreto, a los hospitales para incurables del siglo XIX? Para responder a estas preguntas nos centraremos en un estudio de caso específico: el del Hospital para Mujeres Incurables Nuestro Padre Jesús Nazareno de Madrid. Este hospital, situado en la calle de Amaniel, muy cerca del Cuartel del Conde Duque, fue uno de los hospitales que quedaron bajo control de la Administración Central tras la reforma de la Beneficencia del año 1849, junto al de hombres incurables de la calle de Atocha, el Manicomio de Leganés y el Hospital del Rey, en Toledo. Todos ellos dedicados al cuidado de enfermos con «necesidades permanentes» ${ }^{42}$. La mayoría de sus internos eran calificados como «incurables» y la única terapéutica que se les aplicaba era paliativa, predominando las prácticas «higiénicas» sobre el uso de analgésicos y opiáceos ${ }^{43}$.

El recinto donde se alzaba el hospital había sido, antes de su traslado definitivo en el año 1824, el Colegio de Niñas Monterrey. Contaba con un amplio patio central (ver Imagen 1) alrededor del cual se distribuían las seis salas destinadas a las enfermas. El espacio se presentaba como un «remanso de paz». En un artículo publicado en ABC en el año 1929 se dice de él que era más un asilo que un hospital, y lo describe como un

paraje de calma y quietud; aquí es un grupo de viejecitas que hacen labor, otras que pasean, unas que escuchan la radio, otras que dormitan; ésta refresca las flores del altarcito que tiene junto a la cabecera del lecho, la de más allá, con la mirada vaga y sin pensar en nada, se abanica y se mueve al ritmo de la mecedora; algunas comentan en un grupo y más parecen niñas que ancianas; aquélla, apartada en un rincón, desgrana las cuentas de un rosario... ${ }^{44}$.

42. F. Vidal Galache, "Ser viejo en Madrid. El Hospital de Incurables de Jesús Nazareno y otros centros de asistencia a los ancianos", Espacio, Tiempo y Forma. Serie V, Historia contemporánea, 1993, 367-76; J. M. Zaragoza, "Enfermedad incurable en la España del siglo XIX: el Hospital para Hombres Incurables Nuestra Señora del Carmen”, Dynamis 32, 1, 2012, 141-63.

43. Zaragoza, “Enfermedad incurable...”, op. cit., 156-59.

44. Ramírez Tome, “Los Hospitales de Incurables”, ABC, 27 de julio de 1929. 


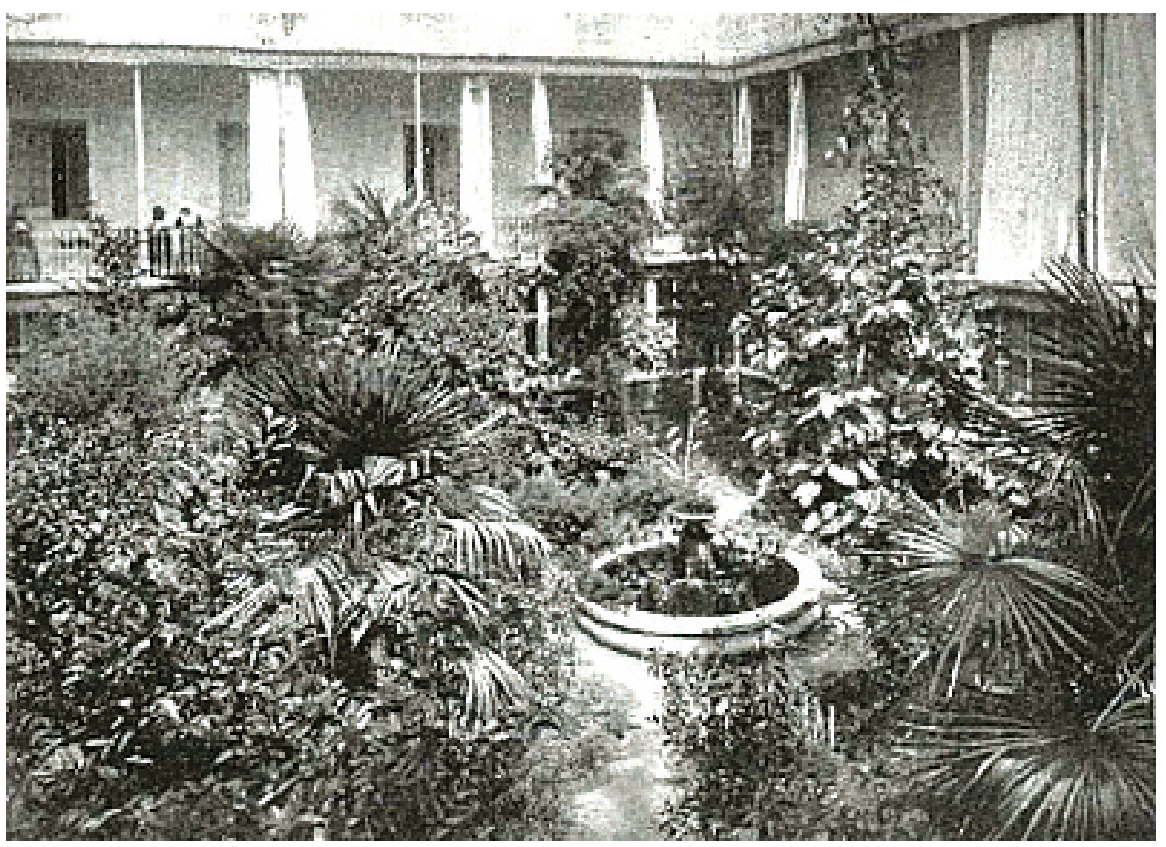

Ilustración 1: Patio del Hospital para Mujeres Incurables, c. $1900 .^{45}$

Lo que observamos en la fotografía del patio del Hospital (Imagen 1) ${ }^{46}$ apunta en esta dirección señalada en el texto. El espacio al que dan las habitaciones de las enfermas es un frondoso jardín en el que apenas podemos vislumbrar un caminito que une la fuente, en el centro de la imagen, con los extremos del patio. En su trabajo sobre los jardines de los hospitales británicos durante los siglos XVIII y XIX, la autora británica Clare Hickman ha señalado la importancia que estos espacios tenían para la terapéutica de diversos tipos de

45. Carlos Osorio, "Caminando por Madrid: El hospital de Jesús Nazareno en Amaniel". En línea en: https://caminandopormadrid.com/el-hospital-de-jesus-nazareno-en-amanie. [Consulta: 5.5.20].

46. La cultura visual es, muchas veces, el único camino que nos queda para estudiar ciertos espacios materiales ya desaparecidos de los que sólo tenemos testimonios visuales, como es este caso. Las relaciones entre cultura visual y material son, en todo caso, mucho más profundas que esta que aquí señalamos. Véase M. Banks y H. Morphy, Rethinking Visual Anthropology, New Haven \& London, 1997 y, en concreto, el capítulo de Françoise Dussart "A body painting in translation", 186-202. En este sentido, somos conscientes de que los resultados pueden ser poco persuasivos. La situación ideal sería, desde luego, poder acudir a fuentes textuales (listados de mobiliario, pertenencias, descripción de las decoraciones de las instalaciones, etc.) que sirvieran para apoyar nuestras conclusiones. Desgraciadamente, en esta ocasión no es posible. No conocemos fuentes textuales, más allá de las citadas en este texto, que describan los interiores de este hospital. Este es el reto que tratamos de enfrentar en este artículo: estudiar espacios desaparecidos a través de las representaciones visuales de los mismos. Esto, que ya consideramos útil por sí mismo, se convierte en necesidad cuando no hay disponibles otras fuentes. 
enfermedades, sobre todo aquellas consideradas crónicas ${ }^{47}$. Relacionado con las prácticas higienistas y con el termalismo, la presencia de jardines en los hospitales ofrecía ventajas que no se limitaban exclusivamente a las posibilidades de hacer más ejercicio físico o al impacto psicológico que parecía tener sobre pacientes con enfermedades mentales, sino que cumplía funciones higiénicas, al ayudar a una mejor ventilación de los pabellones. El espacio interior del hospital de Mujeres Incurables puede entenderse, por tanto, como una herramienta terapéutica, dispuesta para mejorar tanto las condiciones de vida de las enfermas, como para contribuir a la adecuación higiénica del edificio.

Antes de proseguir, debemos señalar sin embargo dos cosas. La primera es que, pese a lo que pudiera escribir Ramírez Tome en 1929, los hospitales de incurables no tuvieron muy buena imagen. Así, Philiph Hauser, en su famoso estudio sobre Madrid publicado en 1903, califica al de hombres en la calle de Atocha como un «foco de insalubridad $»^{48}$ y Concepción Arenal, en un artículo publicado en 1868, denunciaba los abusos que sufrían los internos e internas de ambos hospitales ${ }^{49}$. El segundo aspecto a tener en cuenta tiene que ver, nuevamente, en que aquello que para el $\mathrm{ABC}$ podrían parecer prácticas asilares, en el caso de los hospitales para enfermos incurables durante el periodo estudiado era la única terapéutica posible: mantener a raya las pasiones desbordadas mediante disciplina, rutina y aislamiento ${ }^{50}$.

No ha sido sencillo, en todo caso, encontrar representaciones del interior de ninguno de los dos hospitales de incurables. La que presentamos a continuación se realizó en circunstancias excepcionales, y por tanto debe ser tomada con todas las precauciones (Imagen 2). En la imagen vemos a la Reina Maria Victoria del Pozzo, esposa del Rey de España Amadeo I, y llegada a Madrid el 19 de marzo de 1871. El 24 del mismo mes visitaba el hospital de mujeres incurables. No es la única imagen de este estilo que podemos encontrar, hasta el punto de que casi podemos hablar de una pintura de género, y como tal se encuentra llena de convenciones ${ }^{51}$. Comparémosla con la Imagen 3, que representa a la Reina Victoria visitando a diversos heridos de la tercera guerra anglo-ashanti (1873-1874) en el hospital de Netley

47. C. Hickman, Therapeutic Landscapes: a History of English Hospitals Gardens since 1800, Manchester, 2013.

48. Ph. Hauser, Madrid bajo el punto de vista médico-social, Madrid, 1979; Zaragoza, "Enfermedad incurable ..., op. cit., 150.

49. C. Arenal, “¡Pobres pobres”, La Iberia, 28 de noviembre de 1868.

50. Zaragoza, "Espacios emocionales..., op. cit.

51. Las representaciones de lo que podríamos llamar "reinas visitando enfermos" son numerosísimas, y casi merecerían un estudio aparte. Una búsqueda nada sistematizada en la Wellcome Library ofrece como resultados 37 imágenes, correspondientes al periodo 1800 - 1950, aproximadamente. Encontramos, obviamente, a la reina Victoria pero también a la reina consorte Alexandra (1901-1910), la reina consorte Mary (1910-1936), y la reina consorte Elizabeth (1936-1952). Pero podemos encontrar también imágenes de otras reinas, como la actual reina Elizabeth II, la reina Marie de Rumanía (1914-1927) o, incluso, a la reina Elisabeth de Bélgica (1909-1934) vestida con el uniforma de la Cruz Roja cuidando personalmente a un enfermo. La composición de estas imágenes es siempre muy similar, como veremos en el texto: la reina, rodeada por su séquito, dirige su atención hacia un grupo de enfermos o, incluso, a uno particular. Esto es lo que nos ha empujado a hablar de "pintura de género", partiendo de una definición que tiene más que ver con 
(Southampton). Las similitudes entre ambas son evidentes, tanto en la composición (la reina en el centro, hablando con uno de los enfermos) como en los elementos representados. Ambas pretenden servir a la construcción de la figura pública de ambas reinas. Si la Reina Victoria se presenta ante su pueblo como la madre de Inglaterra, sufriendo por el dolor de sus hijos heridos y llevando luto por los muertos, la italiana se nos muestra como la reina que merece España: joven, hermosa, compasiva y devota. No como la antigua reina. La imagen de Maria Victoria del Pozzo, en definitiva, es parte de la campaña de legitimación del nuevo rey, su esposo, Amadeo I de Saboya, rey de España entre 1870 y 1873.

Ha sido necesario que una reina visitase el establecimiento para encontrar esta imagen, llena de elementos e indicios que compelen a realizar una lectura exhaustiva. El conjunto presentado nos transmite una sensación de calmada serenidad. Resulta obvio decir que la visita de la reina -que no en vano es la absoluta protagonista del grabado-, distorsiona la posible interpretación, pero si nos concentramos en las cuatro mujeres que conforman el grupo situado a la izquierda, si nos aislamos de las miradas adustas de los visitantes, es posible percibir, más allá del respeto debido a la realeza, los rostros tranquilos de las cuatro ancianas. Rostros que encuentran su reflejo en la actitud de la monja al otro lado de la sala, con la que comparten gesto. Un gesto que transmite no sólo respeto a la corona, sino ante todo lo que con absoluta propiedad podemos llamar resignación cristiana. Si al ser admitidas en el hospital se entraba «en otro mundo $»^{52}$, las pasiones de este no tenían lugar en aquel. Esta imagen nos muestra, por tanto, algo más que la visita de una reina, nos muestra el hospital como el espacio en el que se incentiva un determinado estado emocional en sus internas, que se corresponde (al menos teóricamente) con el de las monjas que las atienden, a las que deben emular. El espacio emocional del Hospital de Mujeres Incurables Jesús Nazareno, por tanto, se centra en conseguir que la internas encuentren, a través del sufrimiento y la resignación, la serenidad de aquellos que han sido salvados ${ }^{53}$.

Esta, entendía Concepción Arenal, era la gran ventaja que la enfermedad ofrecía para la salvación del «pobre»: «no sólo tenemos la seguridad de encontrarle a todas horas en su casa, sino la de hallarle mejor dispuesto a escucharnos» ${ }^{54}$. La enfermedad, más allá de que se entienda como resultado de la conducta moral del enfermo, ofrece una oportunidad inestimable para la salvación del alma. Primero, porque aparta al enfermo del «ruido del mundo», que es sustituido por «el silencio de las largas noches [...] tan propio para hacernos entrar en nosotros mismos y oír la voz de la conciencia». Y, en segundo lugar, porque la arrogancia que proporciona la fuerza física es sustituida por «la debilidad del dolor y la disposición a

la teoría literaria (basada en convenciones narrativas compartidas y formas comunes de representación), que con la historia del arte (representaciones de la vida cotidiana o "costumbres").

52. J. de Madrid, Reglamentos y constituciones de la Casa Hospital de Jesús Nazareno para las pobres impedidas é incurables... Madrid, 1803.

53. L. Grenet, Arte de cuidar a los enfermos. Manual teórico práctico para uso de las familias en general y de las enfermeras religiosas en particular, Barcelona, 1903.

54. Concepción Arenal, El visitador del pobre, Madrid, 1946: 127. 


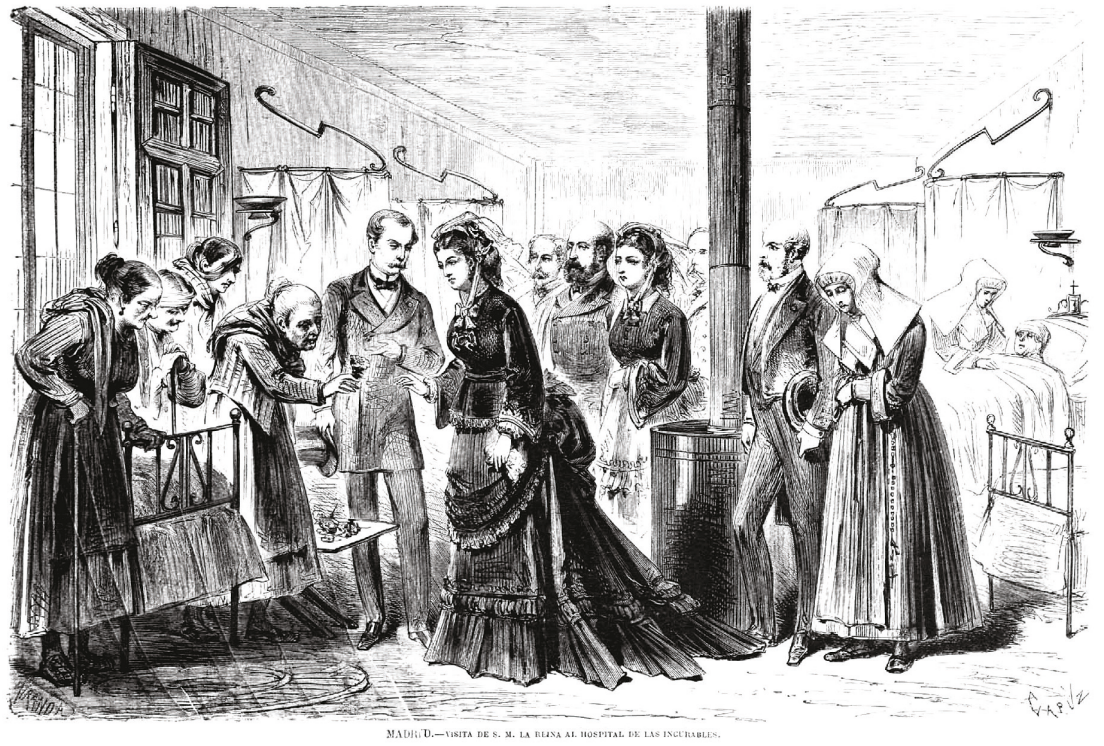

Ilustración 2: Visita de Su Majestad la Reina al Hospital de las Incurables.

Dibujo: Miranda; Grabado: Capuz, La Ilustración Española y Americana, 5 de abril de 1871. Hemeroteca Digital de la Biblioteca Nacional.

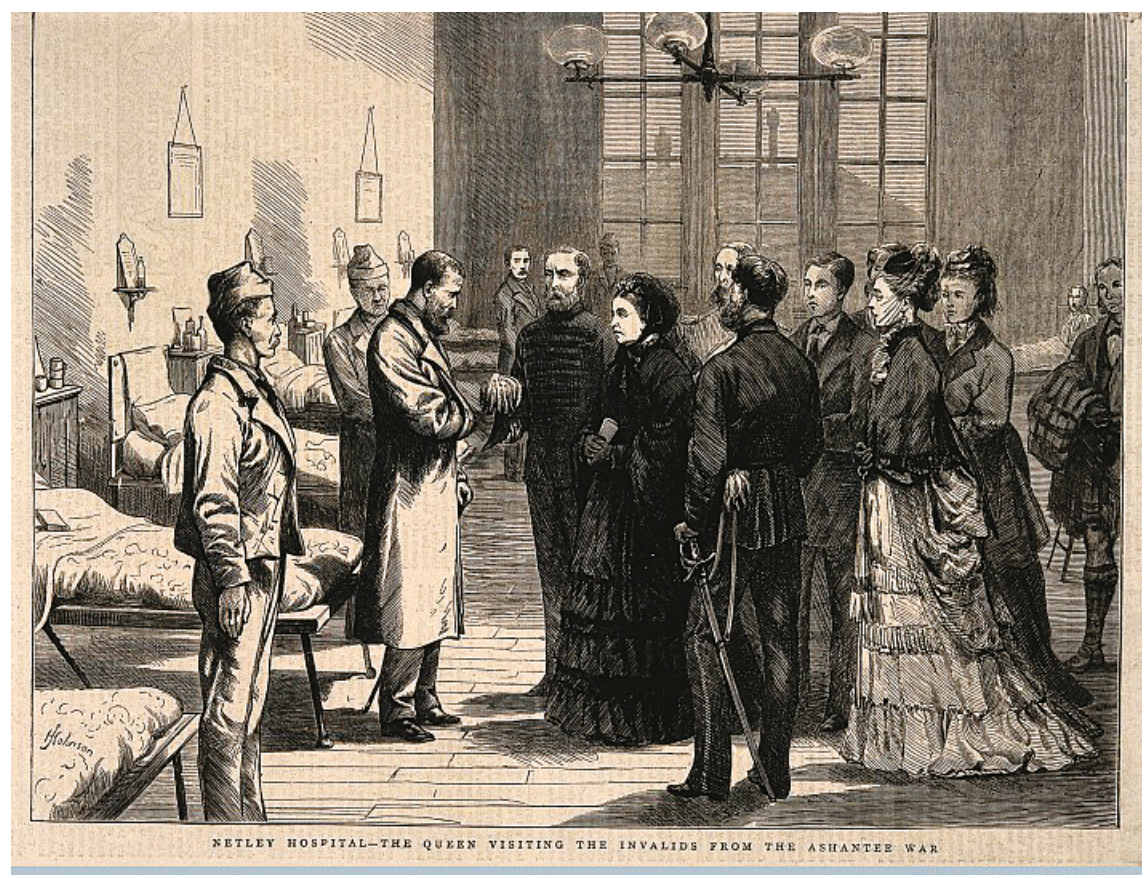

Ilustración 3: Netley Hospital, Hampshire: Queen Victoria visiting invalids from the Ashantee War. Wood engraving by H. Johnson. Wellcome Library. The Graphic, 1874. 
reconocer nuestra miseria» ${ }^{55}$. Que el enfermo sea conducido al hospital, «circunstancia por lo común poco favorable, y que procuraremos evitar», no implica el final de este intento de salvación, sino todo lo contrario. No sólo se deberán renovar los esfuerzos, sino que se buscarán en el mismo hospital «personas caritativas a quienes podamos confiar el secreto de sus faltas ${ }^{56}$. Y si esto es así, si la enfermedad es el momento propicio para la salvación, se debe, únicamente, al papel purificador del dolor: «El dolor purifica lo que está manchado, santifica lo que es bueno y diviniza lo que es santo. Acostumbrémonos, pues, a mirarle como un poderoso auxiliar, que Dios nos envía para la perfección del hombre; como el solo cauterio que puede poner coto a la gangrena de la corrupción humana» ${ }^{57}$.

Las prácticas que contribuyen a crear esta atmósfera de resignación que debía conducir a las enfermas a la salvación religiosa ${ }^{58}$ mediante la interacción entre los sujetos y los objetos en el espacio del hospital son, sin embargo, más difíciles de percibir. El protagonismo de los visitantes niega la representación detallada del espacio. Sin embargo, incluso de esta imagen sesgada es posible extraer ciertos elementos relevantes del campo afectivo. Atendemos en primer lugar al extremo derecho del cuadro. Allí, en un segundo plano, una enferma en el lecho es atendida por una hermana de la Caridad. Se encuentra sobre una cama estrecha, cubierta por sábanas y una manta hasta el cuello, y en la cabeza luce una suerte de vendaje o gorro. La hermana apoya su mano derecha sobre la enferma, y mantiene una conversación con ella, o tal vez está rezando, en lo que parece un intento de confortarla. En la cabecera de la cama encontramos una serie de frascos o vasos y un crucifijo. Separando a esta enferma de su compañera más inmediata, una ligera cortina cuelga de una barra sustentada en la pared (ausente, sin embargo, en la imagen del hospital inglés), conformando grupos de dos camas. Pese a esta pequeña concesión a la intimidad, la sala contiene, como mínimo doce internas. Posiblemente alguna más, pues el artículo de La Ilustración Española en que encontramos la imagen consigna que el hospital contaba con nueve habitaciones, entre las que se repartían $200 \mathrm{camas}^{59}$. No parece haber ninguna mesilla, por lo que los objetos que rodean a la enferma se encuentran fuera de su alcance. Es necesario incorporarse de la cama, algo que no todas serán capaces de hacer. Ese parece ser el caso de la enferma consolada por la hermana: incapaz de moverse de la cama, sin la posibilidad de interaccionar directamente con los objetos que la rodean.

Si abrimos más el foco, reencontramos en la esquina izquierda de la representación al grupo de mujeres coprotagonista de la escena, todas ellas ancianas, excepto, parece, la del fondo. Todas conservan su movilidad, aunque posiblemente reducida por las enfermedades

55. Concepción Arenal, El visitador..., op. cit., 128.

56. Concepción Arenal, El visitador..., op. cit., 134.

57. Concepción Arenal, El visitador..., op. cit., 10.

58. Siguiendo, también aquí, la tradición de las visitadoras de prisiones y de enfermos por la que abogaba Concepción Arenal.

59. Anónimo, "Visita de S.M. la Reina al Hospital de Jesús Nazareno", La Ilustración Española y Americana, 4 de mayo de 1871. 
que solían encontrarse en estos hospitales ${ }^{60}$, y se dedican a algunas de esas pequeñas tareas que se recomendaban para los enfermos. En este caso, la enferma ofrece a la reina una rosa que ella misma, florista de profesión, prepara en la pequeña mesita que vemos a los pies de la cama ${ }^{61}$. Tras ellas, un amplio ventanal o puerta, que deja entrar la luz del atardecer en la sala y que daría al patio que veíamos en la Imagen 1. Todas visten de calle, con largas faldas, chaqueta y chal. El pelo recogido en un moño. No hay alfombras en el suelo, que nos parece de piedra, y la estufa del centro de la sala no parece suficiente para amortiguar el frescor primaveral, de ahí tal vez la presencia de chaquetas y chales.

Son muchos los marcos que se pueden detectar en esta ilustración, incluso si evitamos prestar atención a la reina y su corte. Sin embargo, si atendemos al puramente emocional, encontramos elementos que podíamos esperar (los trabajos manuales, el crucifijo), y otros que pueden sorprendernos más, como la presencia de cortinas separadoras para mantener una cierta sensación de intimidad. Obviamente, hay otros elementos que no apoyan el marco. El principal de ellos sería la presencia de enfermos graves con otros que parecen no estarlo tanto. Pero en general vemos que, en la medida de sus posibilidades (nunca fueron establecimientos especialmente boyantes), los medios físicos tienden a reforzar esa sensación de tranquilidad y resignación. Se puede percibir un cuidado hacia las condiciones de vida de las internas que, asumiendo la difícil situación en que se encuentran, intenta paliar de cierta forma su sufrimiento. Que el único elemento capaz de lograr ese objetivo sea el control de las pasiones, las «prácticas higiénicas» ${ }^{62}$, no deja de ser parte de la realidad de una época en la que, por un lado, la medicina era extremadamente reticente a la administración de los medios necesarios para una eficaz gestión del dolor ${ }^{63} \mathrm{y}$, por otro, las pasiones se seguían considerando el origen principal de muchas enfermedades, tanto mentales como físicas ${ }^{64}$. En los hospitales de incurables, lo que se intentaba, por tanto, no era luchar contra el dolor -una tarea perdida-, sino hacer frente al sufrimiento. Y esto se llevaba a cabo, al menos en nuestro estudio de caso, a través de una interpretación religiosa del mismo que pasaba por su acep-

60. No hemos encontrado datos de ingreso en este hospital, cuya búsqueda continúa. Sin embargo, podemos encontrar similitudes con el Hospital para Hombres Incurables cuyos datos en el periodo arrojan un elevado número de enfermedades que comprometían la movilidad de los enfermos: reumatismo, hernias, hemiplejia, diversos tipos de parálisis y semiparálisis, etc. Zaragoza, "Enfermedad incurable ...”, op. cit., 157. Un resultado similar se obtiene si se consulta, por ejemplo, el trabajo de Charcot sobre enfermedades crónicas: en las enfermas internas en la Salpêtrière, la principal enfermedad era "el reuma": J-M. Charcot, Lecciones Clínicas sobre las Enfermedades de los Viejos y las Enfermedades Crónicas, Madrid, 1883. Vidal Galache, en su artículo pionero, se limita a citar el reglamento de 1803, que señala que sólo se admitía a «mujeres incurables, tullidas o muy ancianas que no se pudieran valer por sí mismas», Vidal Galache, "Ser viejo...", op. cit., 373.

61. Anónimo, "Visita de S.M. la Reina ...", op. cit.

62. Charcot, Lecciones Clínicas ..., op. cit., 234.

63. J. Moscoso, "La medicina del dolor. Una mirada histórica", Métode 71, 2011, 51-59. Javier Moscoso, Historia cultural del dolor, Barcelona, 2011.

64. M. Durand-Fardel, Traité Clinique et Pratique des Maladies des Vieillards, Paris, 1854; P.F. Monlau, Elementos de higiene privada o arte de conservar la salud del individuo, Madrid, 1864, 381. 
tación e integración en un proyecto vital del individuo cuyo único objetivo, a partir de este momento, era lograr la salvación ${ }^{65}$.

\section{Conclusiones}

Como señalábamos en la introducción, el paradigma de las emociones construidas de Lisa Feldman Barrett ofrecía a las humanidades en general, y a la historia en particular, nuevas formas de aproximarse al estudio de las emociones. Si, como señala Barrett, las emociones se componen de aspectos culturales y sociales, si son «un producto del consenso humano» ${ }^{66}$, dejan entonces de ser parte del explanans para ser parte del explanandum: «In this view, emotions do not refer to the things being classified, but rather are classification schemes that people impose on their world during perception ${ }^{67}$. Se produce así un deslizamiento, que nos compele a dejar de hablar de emociones para empezar a hacerlo de experiencia emocional ${ }^{68}$. Es aquí donde la ontología histórica, un programa de investigación desarrollado por Hacking para explicar, precisamente, la aparición de nuevos esquemas clasificatorios, de nuevas categorías aplicadas a humanos y describir su funcionamiento y consecuencias en nuestra experiencia de nosotros mismos, puede desarrollar todo su potencial.

En el presente artículo, hemos intentando demostrarlo estudiando un aspecto muy concreto de nuestra experiencia: la relación con el espacio y la cultura material. Basándonos en diversas fuentes teóricas, hemos desarrollado las herramientas necesarias para analizar, empleando la terminología de Hacking, una «idea en su matriz». La idea, el concepto, era el de enfermo incurable. Más concretamente, en nuestro estudio de caso hemos analizado la experiencia de las mujeres internas en el Hospital para Mujeres Incurables Jesús Nazareno de Madrid. Para ello, hemos llevado a cabo un análisis exhaustivo de las representaciones visuales que han llegado hasta nosotros, intentando comprender cómo la «matriz» material afecta a la experiencia emocional de los sujetos categorizados como «incurables». Conceptos como atmósfera emocional o campo afectivo han demostrado aquí su utilidad. Como en todo análisis parcial, quedan fuera elementos que, en un estudio más amplio, deberán tenerse en cuenta inevitablemente. Cuestiones como el género, la procedencia social, la edad, etc., son aspectos que no pueden dejarse de lado en la descripción de las enfermas y enfermos crónicos. En este artículo nos hemos centrado en un elemento muy concreto (y frecuentemente ignorado) de nuestra experiencia, dejando para ser desarrollado en otros textos otros aspectos que nos ayudarán, sin duda, a entender mejor cómo se constituye la experiencia y su relación con las emociones y las pasiones.

El estudio de las experiencias emocionales que empezamos a vislumbrar es un terreno en el que diversas disciplinas se encuentran, un terreno transdisciplinar en el que aporta-

65. Grenet, Arte de cuidar a los enfermos..., op. cit.

66. Barrett, La vida secreta del cerebro, op. cit., 18.

67. L.F. Barrett, "Are Emotions..., op. cit., 49.

68. Zaragoza, “Investigación filosófica...”, op. cit. 
ciones desde diversas disciplinas contribuyen para incrementar nuestra comprensión de los fenómenos históricos. La ontología histórica de Ian Hacking se presenta como una metodología híbrida, en la que la historia y filosofía colaboran estrechamente en la dilucidación de los aspectos objetivos y subjetivos que posibilitan la aparición de nuevos sujetos y, por tanto, de nuevas formas de experiencias. Las posibilidades a las que nos abre esta colaboración están todavía por explorar. 